EUROPEAN LABORATORY FOR PARTICLE PHYSICS

CERN SL and PPE DIVISIONS

CERN-SL/96-74 (EA)

December 18, 1996

\title{
Beam Spot Position Measurement at the LEP Collider
}

\author{
D. Bourilkov ${ }^{\mathrm{e}}, \mathrm{V}$. Brigljevic ${ }^{\mathrm{e}}, \mathrm{F}$. Filthaut ${ }^{\mathrm{c}}, \mathrm{R}$. Forty ${ }^{\mathrm{a}}$, \\ F. Harris ${ }^{\mathrm{b}}$, R. Hawkings ${ }^{\mathrm{a}}$, G. von Holtey ${ }^{\mathrm{a}}$, E. Migliore ${ }^{\mathrm{d}}$, \\ O. Schneider ${ }^{a}$, E. Vallazza ${ }^{a}$, J. Wenninger ${ }^{\text {a }}$ \\ a CERN, CH-1211 Geneva 23, Switzerland \\ ${ }^{\mathrm{b}}$ Department of Physics, University of Oxford, Keble Road, Oxford OX1 3RH, \\ UK \\ ${ }^{c}$ Carnegie Mellon University, Pittsburgh, PA 15213, USA \\ ${ }^{d}$ Dipartimento di Fisica Sperimentale, Università di Torino and INFN, Via P. \\ Giuria 1, I-10125 Turin, Italy \\ e Eidgenössische Technische Hochschule, ETH Zürich, CH-8093, Switzerland
}

\begin{abstract}
A precise knowledge of the beam spot position is required for many physics topics at LEP2. The movement of the beam spot is studied at LEP1 using beam orbit monitors close to the interaction points and compared with measurements from tracks produced in $e^{+} e^{-}$collisions. The beam orbit monitors are found to follow the beam spot position well, particularly when corrected for movements of nearby quadrupole magnets. Data from the LEP high energy run of November 1995 are also analysed, and projections made for the prospects at LEP2.
\end{abstract}

To be submitted to Nuclear Instruments and Methods 


\section{Introduction}

Experiments at the Large Electron Positron (LEP) collider have studied the properties of long lived particles produced in $Z^{0}$ decays with great success. Analyses of $Z^{0} \rightarrow \mathrm{b} \overline{\mathrm{b}}$ and $Z^{0} \rightarrow \tau^{+} \tau^{-}$events in particular have benefited from precise knowledge of the position of the primary vertex. This allows secondary particles that travel a significant distance before decaying to be clearly separated from those coming directly from the primary vertex. A key ingredient in this knowledge is the measurement of the position and size of the luminous region produced by the colliding $e^{+}$and $e^{-}$beams (the 'beam spot').

Around $\sqrt{s}=91.2 \mathrm{GeV}$ (LEP1), the high rate of tracks from $Z^{0}$ decays allows the position of the beam spot to be followed accurately over time. However at $\sqrt{s} \geq 160 \mathrm{GeV}$ (LEP2) the rate of tracks from $e^{+} e^{-}$collisions is insufficient to follow the beam spot precisely, but a knowledge of its position is still useful, e.g. for $\mathrm{b}$-tagging in the search for $H \rightarrow \mathrm{b} \overline{\mathrm{b}}$. The LEP Beam Orbit Measurement (BOM) system, originally designed as a diagnostic and monitoring tool for accelerator operation, can also be used to measure the beam spot position in the experiments. However, to obtain sufficient precision, it is also necessary to continuously monitor the position of the beam focusing magnets close to the interaction points (IPs) since their positions vary with time.

This paper describes studies of beam spot position measurement performed jointly by accelerator physicists and the LEP experimental groups. An overview of the measurement requirements at LEP2 is first given, followed by descriptions of the BOM hardware, the interpolation method, and the systems for monitoring the magnet positions. Beam spot measurements derived from the BOM system and tracking are then compared, both at LEP1 and the LEP1.5 pilot run at $\sqrt{s}=130-140 \mathrm{GeV}$ in November 1995 . Finally the prospects for LEP2 are discussed. Preliminary results from these studies have already been described in $[1]$.

\section{Beam spot position measurement requirements}

\subsection{Beam spot measurements at LEP1}

The LEP1 luminous region has an approximately Gaussian profile with an RMS size of $120-150 \mu \mathrm{m}$ in $x, 5 \mu \mathrm{m}$ in $y$ and $7 \mathrm{~mm}$ in $z$ respectively ${ }^{1}$. The size has been determined both from machine parameters and from studying the impact parameters of tracks in $Z^{0} \rightarrow \mu^{+} \mu^{-}$events recorded by the LEP experiments. At the highest LEP2 energies, the beam spot size is expected to be similar to that at LEP1 when the low emittance lattice and stronger horizontal focusing are brought into operation.

The movements of the beam spot position over time have been studied extensively at LEP1. The methods are based on fitting tracks measured in the silicon vertex detectors from a number of consecutive events, and deriving a common

\footnotetext{
${ }^{1}$ The coordinate system is defined at each interaction point with positive $x$ towards the centre of the LEP ring, $y$ vertically upwards and $z$ along the electron beam direction. Left and right are defined viewing the detector from the centre of the ring.
} 


\begin{tabular}{|c|c|c|c|}
\hline Experiment & Mean time & \multicolumn{2}{|c|}{ Average resolution } \\
& interval $(\mathrm{mins})$ & $\sigma_{y}^{\mathrm{vtx}}(\mu \mathrm{m})$ & $\sigma_{x}^{\mathrm{vtx}}(\mu \mathrm{m})$ \\
\hline \hline ALEPH & 7 & 9.6 & 16.0 \\
DELPHI & 20 & 4.6 & 17.3 \\
L3 & 20 & 6.5 & 16.2 \\
OPAL & 10 & 8.6 & 18.6 \\
\hline
\end{tabular}

Table 1: Summary of the average resolutions on beam spot positions measured using silicon vertex detectors by the experiments at LEP1. The second column gives the approximate time interval per measurement during the 1995 LEP1 running.

vertex position. Tracks with significant impact parameters (i.e. those from long lived secondary particles) are excluded from the fit. The algorithms typically average over time periods of 7-20 minutes and achieve resolutions below $20 \mu \mathrm{m}$ in $x$ and $10 \mu \mathrm{m}$ in $y$ (see Table 1). Many consecutive independent measurements are thus obtained for each LEP fill (typically 8-15 hours between the beams being brought into collision at the physics energy and being ejected from the machine). Movements of the order of $100 \mu \mathrm{m}$ in both $x$ and $y$ are observed during some fills, both in the form of gradual drifts and sudden jumps in beam spot position.

The main application of beam spot position measurements at LEP1 is in the tagging of long lived secondary particles (primarily b-hadrons). The tagging is done by searching for tracks inconsistent with the primary vertex position, and either determining the probability that all tracks did not come from one vertex, or explicitly reconstructing a secondary vertex. The primary vertex position is determined using the fragmentation tracks in the event, with the beam spot position providing a constraint. Due to the size of the luminous region compared with the track impact parameter resolution (typically $15-200 \mu \mathrm{m}$ depending on momentum), the beam spot constraint is strong in $y$, quite weak in $x$ and negligible in $z$. Studies of LEP1 data indicate that the tagging efficiency is increased by typically $20 \%$ at fixed purity by using the beam spot constraint.

\subsection{Requirements at LEP2}

At LEP2, the main use of beam spot information will again be b-tagging, this time in the search for $H \rightarrow \mathrm{b} \overline{\mathrm{b}}$. The power of the beam spot information can be quantified by the decrease in integrated luminosity $\mathcal{L}_{\text {int }}$ required to discover a particular Higgs. Simulation studies of signals and expected backgrounds have been performed to determine $\mathcal{L}_{\text {int }}$ for various beam spot resolutions [1]. These studies show decreases of $10-20 \%$ in $\mathcal{L}_{\text {int }}$ with a beam spot resolution of $50 \mu \mathrm{m}$ in $x$ and $20 \mu \mathrm{m}$ in $y$, compared to no beam spot information. No significant advantage was found using higher precision measurements, and resolution 2-3 times worse still gives some improvement. However, being conservative and bearing in mind the likely small number of candidate events, a resolution of $20 \mu \mathrm{m}$ in $y$ and $50 \mu \mathrm{m}$ in $x$ is desirable. 


\subsection{Measurements from two-photon events}

The cross section for $\gamma \gamma$ events increases logarithmically with energy, and will be the dominant source of tracks at LEP2. Unfortunately, the tracks from $\gamma \gamma$ events have very forward peaked angular distributions, and consequently low transverse momentum $\left(p_{T}\right)$, and are difficult to measure accurately. Despite these problems, a significant number of tracks are produced in the experiments' central tracking systems, which will be useful for beam spot determination.

Simulation studies have been performed and cross checked with $\gamma \gamma$ events at LEP1, indicating that measurements of the required accuracy can be performed on a time scale of 10-30 minutes at a luminosity of $3 \times 10^{31} \mathrm{~cm}^{-2} \mathrm{~s}^{-1}$ [1]. The accuracy depends strongly on the angular acceptance and minimum track $p_{T}$ used in the measurements, and hence also on the background and trigger conditions (see Section 7). However, the beam spot is known to move around on time scales of a few minutes so another independent method of following its motion is desirable.

\section{The LEP BOM system}

The LEP Beam Orbit Measurement system (BOM) consists of 504 Beam Position Monitors (BPM) and 40 electronics processing stations [2, 3]. Each BPM measures the position of the centre of gravity of the beam charge distribution in both planes (the 'beam position') using four capacitive button electrodes. The BPM is mechanically mounted on a quadrupole and aligned on the quadrupole magnetic axis with an RMS precision of $0.2 \mathrm{~mm}$. A total of 240 BPMs are installed in the LEP arcs on the vertically focusing quadrupoles. The remaining 264 BPMs are mounted on quadrupoles in the straight sections around the IPs. The processing electronics is located in 24 shielded areas. The front-end electronics and acquisition is auto-triggered.

A special 'wide band' electronics system is used for the 56 monitors located close to an IP, where the time between the passage of bunches from the counterrotating $e^{+}$and $e^{-}$beams is shorter than $600 \mathrm{~ns}$. Each button electrode is processed separately by a low pass integrator. The signal amplification is variable and can be switched by high precision relays. The pulses are sampled by an integrate/hold circuit whose output is digitised by a 12-bit ADC. A fast analogue gate allows selection of the $e^{+}$or $e^{-}$beam, but only one particle type can be measured at a time. Since the introduction of bunch trains an external gating has been implemented which allows the selection of a single bunch family. The precise calibration of the gain settings is the main limitation of the wide band BPMs. Imperfections in the calibrations lead to drifts and jumps of the measured positions when the amplitudes of the electrode signals are not in the optimum range of the electronics and when the gains are switched. The magnitude of these systematic effects reaches about $200 \mu \mathrm{m}$ in both planes (at the BPM).

The data supplied to the experiments from the wide band BPMs are averaged over 112 position measurements. Only every second turn is measured such that the average samples exactly one $50 \mathrm{~Hz}$ period to suppress noise, and one orbit is recorded every $40 \mathrm{~s}$.

The BOM system performance is presently well within its specifications for 
the usual accelerator diagnostics. The measurement of the absolute beam spot position at the IP is however a challenge, particularly since it requires very good long term stability. Its performance for measuring beam parameters at the IP on short time scales has been shown to be excellent. The algorithm described below to reconstruct the IP parameters has also been used to detect the electromagnetic interaction of the two beams at the IP and to optimize the vertical beam overlap with an accuracy of better than $1 \mu \mathrm{m}$ [4]. To track the beam spot positions over periods of days or weeks, it may be necessary to renormalize the BOM measurements to vertex detector measurements for each fill or group of fills. In this way, the systematic effects caused by drifts in the gain calibrations discussed above can be eliminated.

\section{Beam Position Interpolation}

The beam positions at the IP can be obtained by interpolating position measurements from nearby BPMs using the transport matrices of the beam optics. A 4-dimensional formalism is used to take into account the large solenoids of the LEP experiments, which couple the horizontal and vertical planes, and the skew quadrupoles used to correct this coupling. The position vector of the beam at a particular location is given by:

$$
\mathbf{v}=\left(\begin{array}{c}
x \\
x^{\prime} \\
y \\
y^{\prime}
\end{array}\right)
$$

where $x, x^{\prime}$ and $y, y^{\prime}$ are the beam positions and angles in the horizontal and vertical plane. Since BPMs cannot measure the beam angle, the beam position measurements of two BPMs (labelled $a$ and $b$ ) have to be combined to extract the four coordinates $\mathbf{v}^{*}$ of the beam at the IP, which can be expressed as:

$$
\mathbf{v}^{*}=\mathbf{M}^{a} \mathbf{v}^{a}+\sum_{i} \mathbf{M}^{i} \theta^{i}=\mathbf{M}^{b} \mathbf{v}^{b}+\sum_{j} \mathbf{M}^{j} \theta^{j}
$$

where $\mathbf{M}^{a}$ and $\mathbf{M}^{b}$ are the transport matrices from the BPMs to the IP, and $\theta^{i}$ are the deflections due to orbit corrector magnets and electrostatic devices seen by the beam between the BPM and the IP. For orbit correctors $\theta^{i}$ is identical for the $e^{+}$and $e^{-}$beams while for electrostatic devices the deflections have the same magnitude but the opposite sign. The matrix $\mathbf{M}^{i}$ is the transport matrix from the location of the deflection $\theta^{i}$ to the IP. All transport matrices have been calculated with the MAD simulation program [5]. Such a formalism based on linear optics can be used since no non-linear elements are installed close to the IP. Using the inverse matrix $\mathbf{N}=\mathbf{M}^{-\mathbf{1}}$ :

$$
\begin{array}{ll}
\mathbf{N}^{a} \mathbf{v}^{*}=\mathbf{v}^{a}+\mathbf{w}^{a}, & \mathbf{w}^{a}=\mathbf{N}^{a} \sum_{i} \mathbf{M}^{i} \theta^{i} \\
\mathbf{N}^{b} \mathbf{v}^{*}=\mathbf{v}^{b}+\mathbf{w}^{b}, & \mathbf{w}^{b}=\mathbf{N}^{b} \sum_{j} \mathbf{M}^{j} \theta^{j}
\end{array}
$$


We can now define the auxiliary vectors $\mathbf{u}$ and $\mathbf{r}$ :

$$
\mathbf{u}=\left(\begin{array}{c}
x^{a} \\
y^{a} \\
x^{b} \\
y^{b}
\end{array}\right)=\left(\begin{array}{c}
v_{(1)}^{a} \\
v_{(3)}^{a} \\
v_{(1)}^{b} \\
v_{(3)}^{b}
\end{array}\right) \quad \mathbf{r}=\left(\begin{array}{c}
w_{(1)}^{a} \\
w_{(3)}^{a} \\
w_{(1)}^{b} \\
w_{(3)}^{b}
\end{array}\right)
$$

and the matrix $\mathbf{W}$ :

$$
\mathbf{W}=\left(\begin{array}{lll}
N_{(11)}^{a} & \cdots & N_{(14)}^{a} \\
N_{(31)}^{a} & \cdots & N_{(34)}^{a} \\
N_{(11)}^{b} & \cdots & N_{(14)}^{b} \\
N_{(31)}^{b} & \cdots & N_{(34)}^{b}
\end{array}\right)
$$

where $N_{(i j)}$ stands for the element in row $i$ and column $j$ of the matrix $\mathbf{N}, v_{(i)}$ is the component $i$ of the vector $\mathbf{v}$, and similarly for $\mathbf{w}$. The vector $\mathbf{u}$ is completely determined by the BPM measurements, and $\mathbf{r}$ can be evaluated from the known deflections. The beam position at the IP $\mathbf{v}^{*}$ can now be determined as:

$$
\mathbf{v}^{*}=\mathbf{W}^{-1}(\mathbf{u}+\mathbf{r})
$$

The two BPMs that have been chosen for the interpolation are mounted on the QS0 and QS4 quadrupoles (see Fig. 1). Due to the layout of the quadrupoles near the IP, changes in the vertical beam angle at the IP produce large beam position movements at the QS0 BPM (BPM $a$ ). Conversely, changes in the vertical beam position at the IP produce large movements at the QS4 BPM (BPM $b$ ). This can be seen from the following numerical example of $\mathbf{W}^{-\mathbf{1}}$ for IP6 (the other IPs are similar). Displacements are measured in mm and angles in mrad:

$$
\left(\begin{array}{c}
x^{*} \\
x^{\prime} \\
y^{*} \\
y^{\prime *}
\end{array}\right)=\left(\begin{array}{rrrr}
0.5839 & 0.0001 & -0.4766 & 0.0002 \\
-0.0149 & 0.0010 & -0.0826 & 0.0000 \\
0.0025 & -0.0196 & -0.0020 & -0.0374 \\
-0.0001 & -0.2339 & -0.0004 & -0.0049
\end{array}\right) \cdot\left(\begin{array}{c}
x^{a}+w_{(1)}^{a} \\
y^{a}+w_{(3)}^{a} \\
x^{b}+w_{(1)}^{b} \\
y^{b}+w_{(3)}^{b}
\end{array}\right)
$$

Using this interpolation method, an independent estimate for the beam position $\mathbf{v}^{*}$ can be obtained from each side of the IP. However, misalignments of the quadrupoles are not taken into account. Displaced quadrupoles produce additional dipole fields which distort the particle orbit. These deflections are compensated as well as possible with orbit corrector dipoles. The quality of the orbit correction is however limited by the number of corrector magnets and the accuracy of the alignment of each BPM relative to the quadrupole axis. The effect on $\mathbf{v}^{*}$ of a misaligned quadrupole located between the IP and the BPMs used for the interpolation is identical to the effect of a corrector. As long as the misalignment does not change with time, it will only shift the absolute value of $\mathbf{v}^{*}$ calculated from Equ. 7.

The superconducting low-beta quadrupoles (QS0s) installed at a distance of $4.7 \mathrm{~m}$ on each side of the IP are the strongest quadrupoles of LEP. They are used to focus the beam in the vertical plane at the IP and the vertical orbit of LEP is very sensitive to movements of these quadrupoles. Large vertical orbit drifts have been traced back to QS0 movements of a few tens of $\mu \mathrm{m}$. The quality of the orbit can be characterised by $\Sigma$, the RMS spread of the BPM position measurements 


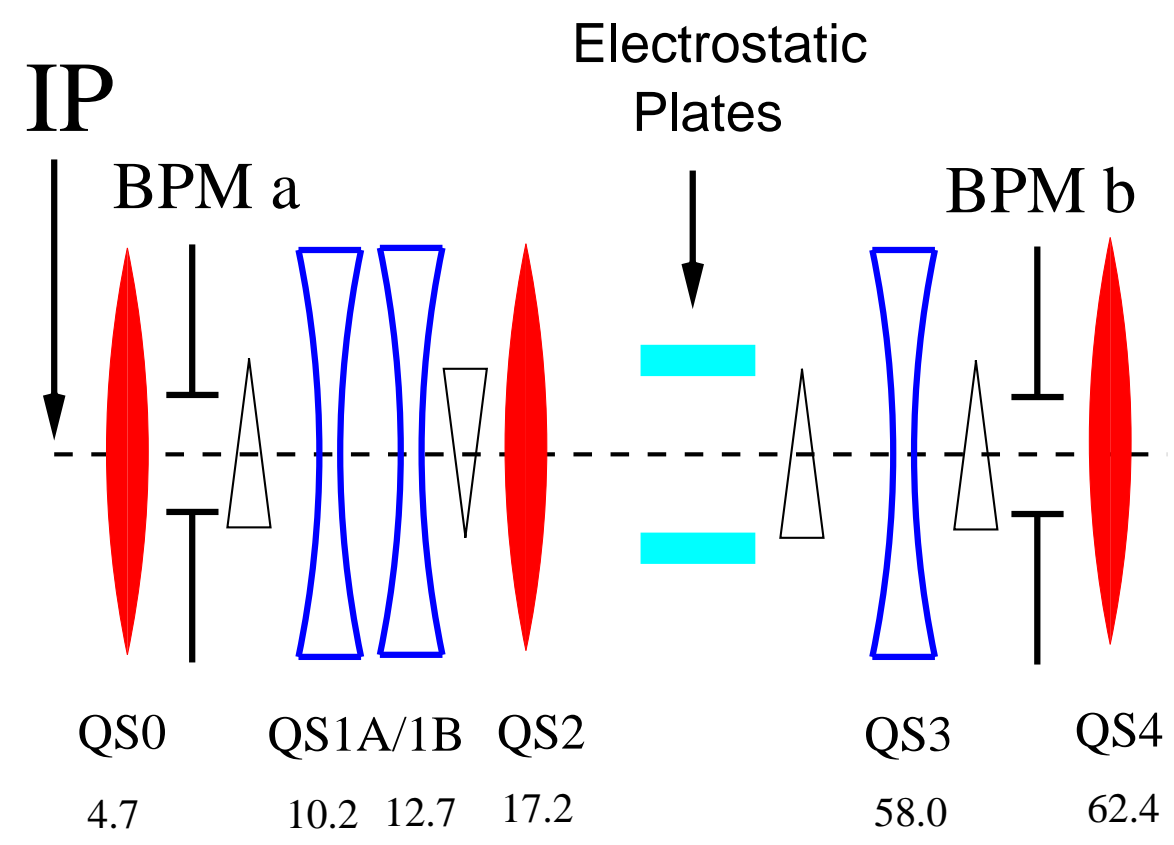

Figure 1: Position of the quadrupoles and the BOM pickups (BPMs) on one side of an IP region. Focusing lenses correspond to vertically focusing quadrupoles, while defocusing lenses correspond to horizontally focusing quadrupoles. The vertical corrector magnets are represented by upright triangles and the horizontal corrector magnet by an inverted triangle. The electrostatic plates are used to separate the beams at the IP. The BPMs next to quadrupoles QS0 and QS4 labelled $a$ and $b$ are used for the interpolation. The machine layout is symmetric around the IP and the beam spot position can be determined independently from each side of the IP. The scale is only approximate - the numbers indicated below the quadrupole names give the longitudinal distances from the IP in metres.

around the entire LEP ring. For a vertical displacement of the QS0s by $y_{L}\left(y_{R}\right)$ on the left (right) side of the IP, the change of the RMS of the vertical closed orbit $\Sigma_{y}$ is given by:

$$
\delta \Sigma_{y} \simeq \sqrt{\left(C_{\Delta}\left(y_{L}-y_{R}\right)\right)^{2}+\left(C_{\|}\left(y_{L}+y_{R}\right) / 2\right)^{2}}
$$

For the usual LEP physics optics, $C_{\Delta} \approx 40$ and $C_{\|} \approx 8.0$ (the analogous numbers for the horizontal plane are 3.7 and 4.0). The vertical orbit is therefore mainly sensitive to differential movements of the QS0s $\left(y_{L}-y_{R} \neq 0\right)$, but it is not possible to determine which quadrupole is moving. Parallel movements $\left(y_{L}+y_{R} \neq 0\right)$ are very difficult to detect with reasonable accuracy. Their effect on the orbit is small because the deflections due to the two QS0 quadrupoles compensate each other very accurately. On the other hand, the change of the vertical beam spot position at the IP, $\Delta y^{*}$, is only affected by the average displacement:

$$
\Delta y^{*}=\alpha\left(y_{L}+y_{R}\right) / 2
$$

where $\alpha$ is calculated from simulation to be approximately 1.4 for the usual LEP optics. Thus the movements in beam spot position induced by QS0 motion are of the same order as the movements of the magnets themselves. 
The problem of the QS0 movement can be overcome provided that a measurement of the vertical position of each QS0 is available. With such information, the beam spot position calculated from the BPMs can be corrected using Equ. 10. A correction can be applied independently for each side of the IP with $\left(y_{L}+y_{R}\right) / 2$ replaced by $y_{L}$ or $y_{R}$ for the left or right side interpolation.

In addition to uncertainties due to quadrupole movements, the accuracy of the interpolation method is limited by the systematic errors on the BPM measurements and uncertainties in the beam optics. Focusing errors of the QS0s are the most important source of optical errors for the LEP collider. These errors are mainly due to uncertainties in the magnetic field calibration curves and beam energy. They lead to errors on the beam spot position of about $\pm 1 \mu \mathrm{m}$. The BPM measurement errors of up to $200 \mu \mathrm{m}$, due to gain calibration problems, lead to systematic errors at the IP of the order of $\pm 5 \mu \mathrm{m}$ and $\pm 100 \mu \mathrm{m}$ in the vertical and horizontal planes respectively. The difference is due to the much stronger focusing of the beam into the IP in the vertical plane.

\section{$5 \quad$ QS0 magnet position monitoring}

The above analysis indicates that monitoring of the QS0 magnet movements is important for the interpolation of the BOM beam spot position data. In addition, analysis of the orbit corrections required during stable physics running indicates that most orbit deviations are caused by movements of the QS0s. To study these effects and eventually correct for them, monitoring systems have been installed at some of the IPs.

\subsection{Potentiometer Probes}

In IP4 (ALEPH) and IP8 (DELPHI) the QS0 magnet positions have been monitored by means of electro-mechanical position sensors, designed to operate in a high magnetic field environment [6]. Such devices were first installed at IP8 during 1994 to monitor the position of the luminosity calorimeters attached to the QS0s. In 1995, their readout and configuration at IP8 was changed specifically for beam spot position studies and sensors were also installed at IP4.

Each device consists of a $32 \mathrm{~mm}$ long body traversed by a metal pin. One end of

the pin is spring loaded and mechanically connected to the variable voltage point of a $10 \mathrm{k} \Omega$ potentiometer integrated into the body. The other end is arranged to touch a reference surface. Movements of the reference surface therefore produce a change of resistance in the potentiometer, which is digitized and read out by the slow controls system of the experiment.

The setup of the probes at the different IPs reflects the specific layout of each experiment. In IP4 the probes were installed on horizontal bars parallel to the beam line and rigidly fixed to the QS0 support girder at approximately $38 \mathrm{~cm}$ from the end closest to the IP. The reference surfaces probed by the pins were attached to LCAL, one of the ALEPH luminometers, which is assumed not to move with respect to the ALEPH tracking system. Four probes were installed on the right side of the experiment, measuring movements in $x, y, z$ and a second independent measurement of $y$, and two on the left side measuring $x$ and $y$. 


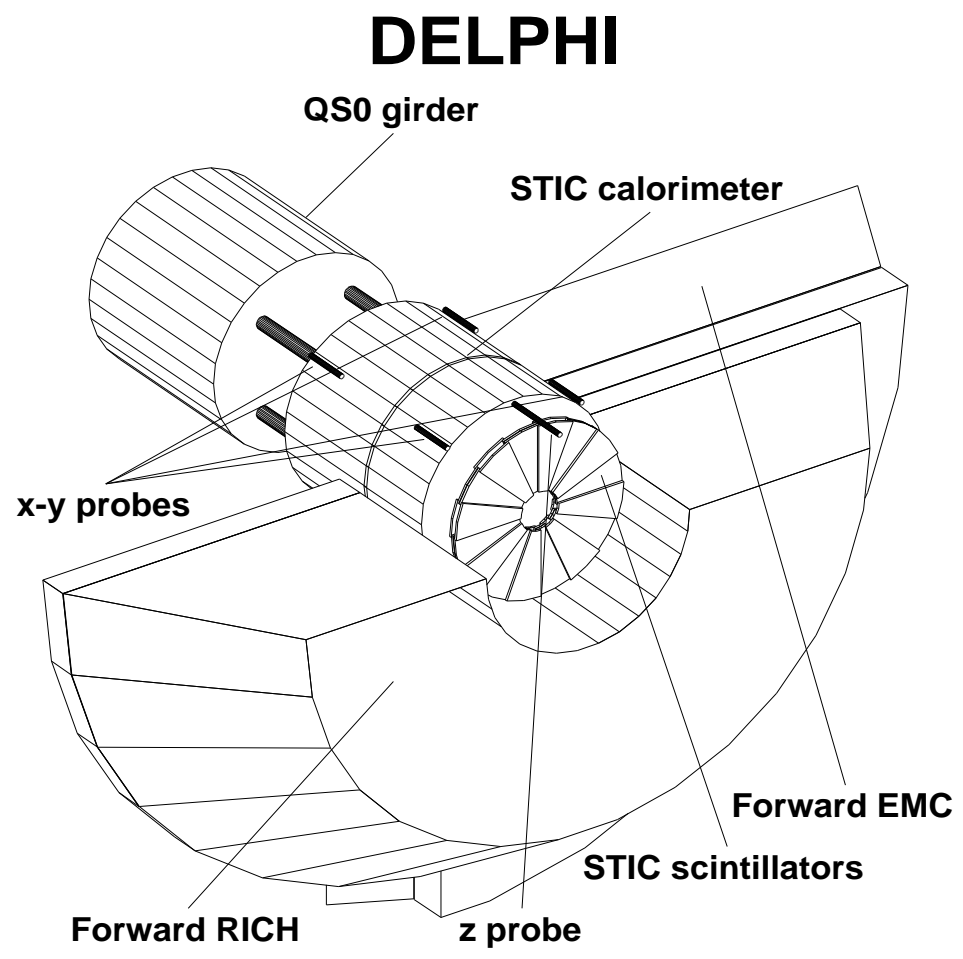

Figure 2: Simplified view of the potentiometer probe layout at IP8 (DELPHI). The STIC (of outer diameter $42 \mathrm{~cm}$ ) is supported by four stainless steel rods attached to the QS0 girder. The figure (not to scale) shows the positions of the $x$ $y$ probes measuring the displacement of the STIC relative to the inner wall of the tube supporting the forward RICH and forward EMC. The $z$ probe consists of a horizontal rod (not to scale) fixed to the STIC calorimeter with the potentiometer mounted at the end touching the TPC laser support.

In IP8 the probes were mounted on the external surface of the STIC, the DELPHI luminometer, which is fixed to the girder which supports the QS0. The reference surface was the inner wall of the tube supporting the forward Ring Imaging Cerenkov detector (RICH) and the Forward Electro Magnetic Calorimeter (FEMC), as shown in Fig. 2. Nine probes were mounted on each side of the interaction point: eight probes situated on two different planes transverse to the beam line (at $40 \mathrm{~cm}$ and $121 \mathrm{~cm}$ from the end of the QS0 girder) at an angle of $45^{\circ}$ to the plane of the machine (in Fig. 2 the four upper probes are shown), and one probe for measuring longitudinal $(z)$ displacements with respect to a fixed vertical plane (the TPC lasers support).

In both experiments a calibration was performed by inserting plates of varying thickness between the probe and the reference surface, and no significant deviations from linearity were observed. The redundancy of the probes was used to estimate the real accuracy of the system by studying the residuals of two independent measurements of the same coordinate. The accuracy of a single probe of 


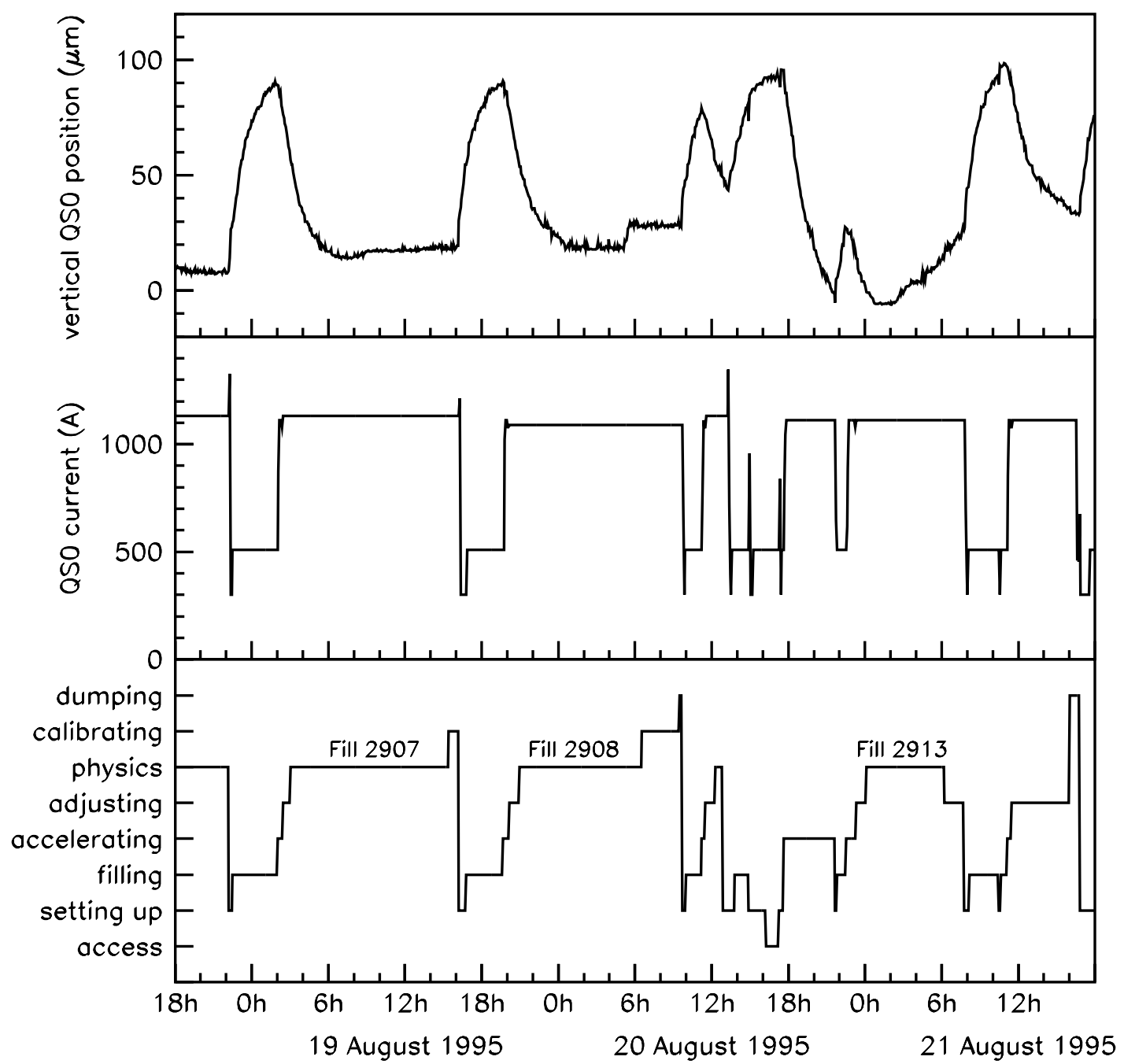

Figure 3: Vertical position of the right side QS0 magnet at IP4, as measured by the ALEPH potentiometer probes, for three consecutive days of data taking. The magnet current and the status of LEP are also shown for the same time interval.

about $2 \mu \mathrm{m}$ was further improved to $\sim 1.5 \mu \mathrm{m}$ by combining the measurements of more probes on the same side of the interaction point.

The measured QS0 vertical positions show a correlation with the machine operation: the magnets move upwards as soon as their currents are set to $\sim 500 \mathrm{~A}$ (20 GeV beam energy setting) for LEP filling, and then downwards again when the currents are ramped to $\sim 1100 \mathrm{~A}$ ( $45 \mathrm{GeV}$ setting) during acceleration (see Fig. 3). These movements appear to be temperature related, being caused by thermal expansion of the QS0 support structure due to the heating effect caused by the currents flowing in the QS0 bus bars. The time constant of the movements is rather long ( $\sim 1-2$ hours) so the quadrupoles are still moving when the beams start 


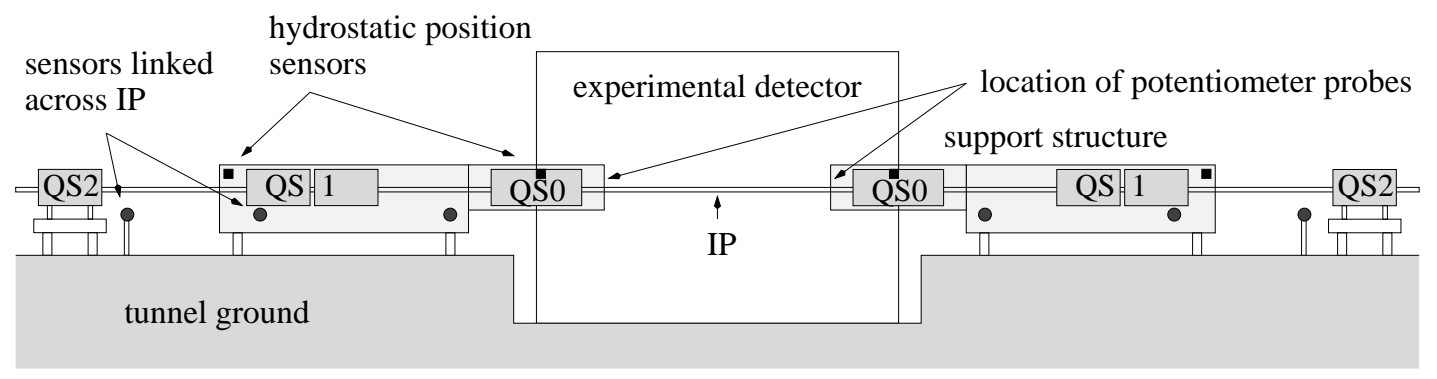

Figure 4: Schematic drawing (not to scale) showing the layout of QS0 magnets, pin potentiometer probes and hydrostatic sensors at IP8

colliding and physics data taking starts. Upward movement is also seen during the latter part of some fills. Movements of the QS0s in the horizontal plane, directly correlated with the magnet currents but with a shorter time constant, were also observed.

The typical amplitude of the vertical movements at IP4 is around $30 \mu \mathrm{m}$ (left side) and $60 \mu \mathrm{m}$ (right side), while it is only about $20 \mu \mathrm{m}$ in IP8. This difference has been interpreted as due to the different type of QS0 magnets installed in the two IPs. For the 1995 running, IPs 2, 6 and 8 were equipped with new QS0 magnets designed for LEP2, whilst IP4 still used an older, heavier design, which operates at a $40 \%$ higher current for the same focusing strength. These magnets have also been upgraded for LEP2.

\subsection{LEP Position Measuring Systems}

In 1994, to aid the survey alignment in IP8, a system was installed to measure the tilt of the QS0 magnets. The system is based on the measurement of differential hydrostatic pressure between 2 communicating pots containing water. One pot is mounted on the QS0 and the other mounted on the supporting girder beam some $9.25 \mathrm{~m}$ further away from the IP. The system thus measures movement of the magnet relative to the other end of its supporting girder. Another system, consisting of sensors linked between the two sides of the interaction point, is used to measure movements of one side of the tunnel relative to the other. The complete system, also including the pin potentiometer sensors, is shown in Fig. 4. A somewhat different system was also installed in IP2 (L3), however this did not directly measure the QS0 positions and was not used in this study.

The system was calibrated for slope measurements by rotating the assembly about a point some $3.5 \mathrm{~m}$ from the QS0 using a tilting motor. The overall resolution is limited in principle by the resolution of the electronics measuring the differential pressure, and also by the temperature. The overall resolution is estimated to be around $5 \mu \mathrm{m}$, and only the vertical coordinate can be measured. The results from this system have been compared to the vertical measurements from the DELPHI potentiometer probes, and have been found to agree well. 


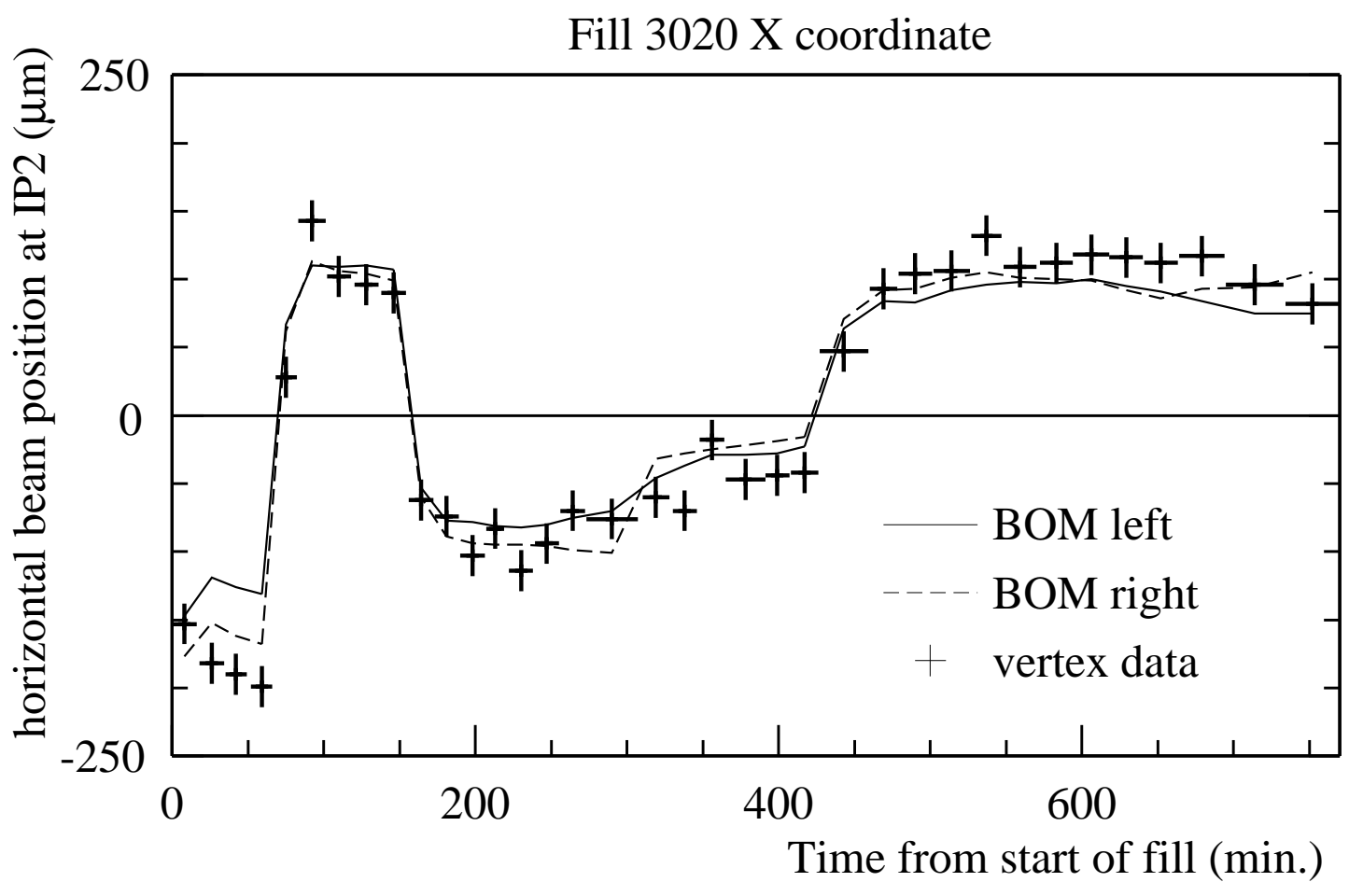

Figure 5: Horizontal beam spot measurements at IP2 (L3) for one fill, showing vertex detector and BOM measurements.

\section{System performance at LEP1}

The performance of the BOM system has been studied during the 1995 LEP1 energy scan period, from 13th August to 5th October. The data was divided into short 'chunks', corresponding to the time required to derive one beam spot measurement from the tracking and vertex detectors (see Table 1). These beam spot position measurements were then compared to those derived from the BOM system and QS0 monitoring in each chunk. Each experiment analysed the data in a similar way; however due to various timing problems with the BOM system, and the different QS0 monitoring systems, the exact set of LEP fills which were analysed differs between experiments.

Some typical data used for this study is shown in Figs. 5 and 6 . Figure 5 compares horizontal beam spot measurements derived from BOM and vertex data for one fill in L3, and Fig. 6 shows similar data in the vertical plane from OPAL. Both these fills show rather large beam spot movements which are correctly tracked by the BOM system.

\subsection{Resolution of different beam spot position estimates}

In order to quantify the beam spot resolutions achieved by the BOM system, and the improvements brought by monitoring the QS0 positions, the following procedure was adopted. Let $y$ be the measurement of the vertical beam spot position from the method under test. The 'per period' resolution $\sigma_{y}^{\mathrm{p}}$ of $y$ is defined 


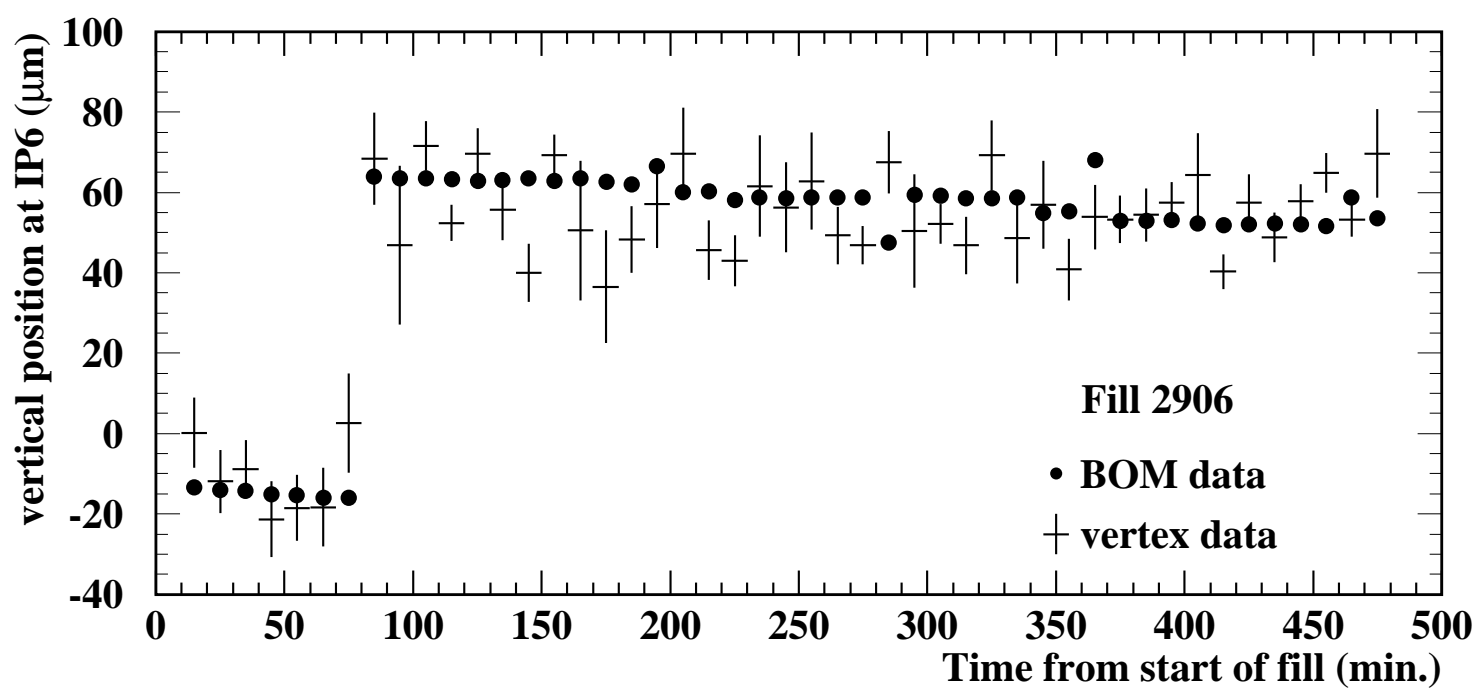

Figure 6: Vertical beam spot measurements at IP6 (OPAL) for one fill, showing vertex detector and $\mathrm{BOM}$ measurements.

as:

$$
\sigma_{y}^{\mathrm{p}}=\sqrt{\operatorname{RMS}^{2}\left(y-y^{\mathrm{vtx}}\right)-\left(\sigma_{y}^{\mathrm{vtx}}\right)^{2}}
$$

where $y^{\mathrm{vtx}}$ is the corresponding measurement using the vertex detector and $\sigma_{y}^{\mathrm{vtx}}$ is the mean error on $y^{\mathrm{vtx}}$ (taken from Table 1). The RMS is computed over all available measurements from the data taking period of 58 fills.

As well as the intrinsic resolution of the measurement $y$, the 'per period' resolution $\sigma_{y}^{\mathrm{p}}$ is also sensitive to long term changes in the absolute offset between $y$ and $y^{\mathrm{vtx}}$. It is therefore also of interest to consider the 'fill by fill' resolution $\sigma_{y}^{\mathrm{f}}$, defined as:

$$
\sigma_{y}^{\mathrm{f}}=\sqrt{\operatorname{RMS}^{2}\left(y-y^{\mathrm{vtx}}-y^{\mathrm{off}}\right)-\left(\sigma_{y}^{\mathrm{vtx}}\right)^{2}}
$$

where $y^{\text {off }}$ is a fill-dependent offset estimated as the average value of $y-y^{\text {vtx }}$ over a given fill. In this way, the contributions from long term changes in the BOM-vertex offsets are removed.

Using this formalism, three different measurements of $y$ were considered:

1. Constant assumption which corresponds to $y=$ constant, (i.e. it is assumed that the beam spot is perfectly stable and that no measurement is needed);

2. BOM alone measurement, which corresponds to $y=y^{*}$, the beam spot position extrapolated from the BOM measurements (for these comparisons, the measurements from $e^{+}$and $e^{-}$beams from each side of the IP were averaged); 


\begin{tabular}{|c|c|c|c|c|c|}
\hline \multirow{2}{*}{ Experiment } & \multirow{2}{*}{ Measurement } & \multicolumn{4}{|c|}{ Resolutions on beam spot position } \\
& & $\sigma_{y}^{\mathrm{p}}(\mu \mathrm{m})$ & $\sigma_{y}^{\mathrm{f}}(\mu \mathrm{m})$ & $\sigma_{x}^{\mathrm{p}}(\mu \mathrm{m})$ & $\sigma_{x}^{\mathrm{f}}(\mu \mathrm{m})$ \\
\hline \hline & 'constant' & $24.4 \pm 0.4$ & $9.2 \pm 0.2$ & $75.6 \pm 1.0$ & $35.9 \pm 0.5$ \\
ALEPH & BOM & $14.8 \pm 0.3$ & $8.5 \pm 0.2$ & $39.9 \pm 0.6$ & $24.8 \pm 0.4$ \\
(IP4) & BOM+QS0 & $7.2 \pm 0.3$ & $3.8 \pm 0.3$ & $39.9 \pm 0.6$ & $24.8 \pm 0.4$ \\
\hline & 'constant' & $16.8 \pm 0.6$ & $8.3 \pm 0.3$ & $42.2 \pm 1.4$ & $17.4 \pm 0.6$ \\
DELPHI & BOM & $12.6 \pm 0.4$ & $8.0 \pm 0.3$ & $33.5 \pm 1.1$ & $22.0 \pm 0.8$ \\
(IP8) & BOM+QS0 & $10.2 \pm 0.3$ & $7.0 \pm 0.2$ & $33.5 \pm 0.1$ & $22.0 \pm 0.8$ \\
\hline L3 & 'constant' & - & $7.2 \pm 0.3$ & - & $48.8 \pm 1.3$ \\
(IP2) & BOM & - & $7.1 \pm 0.3$ & - & $24.7 \pm 0.7$ \\
\hline OPAL & 'constant' & $28.7 \pm 0.5$ & $6.5 \pm 0.5$ & $74.1 \pm 2.0$ & $20.9 \pm 1.0$ \\
(IP6) & BOM & $10.3 \pm 0.4$ & $7.4 \pm 0.1$ & $17.9 \pm 0.8$ & $17.3 \pm 0.7$ \\
\hline
\end{tabular}

Table 2: Summary of beam spot resolutions measured by the experiments (see text). Errors given are statistical.

\begin{tabular}{|c|c|c|}
\hline Experiment & \multicolumn{2}{|c|}{ Value of $\alpha$ which minimises: } \\
& $\sigma_{y}^{\mathrm{p}}$ & $\sigma_{y}^{\mathrm{f}}$ \\
\hline \hline ALEPH & $1.16 \pm 0.02$ & $1.37 \pm 0.03$ \\
DELPHI & $1.31 \pm 0.09$ & $1.33 \pm 0.09$ \\
\hline
\end{tabular}

Table 3: Values of $\alpha$ which minimise $\sigma_{y}^{\mathrm{p}}$ and $\sigma_{y}^{\mathrm{f}}$ for $y=y^{*}+\alpha\left(y_{L}+y_{R}\right) / 2$. These numbers are not directly comparable because the QS0 magnets and the way their position is measured are not identical in the two experiments.

3. BOM+QS0 measurement, which corresponds to $y=y^{*}+\alpha\left(y_{L}+y_{R}\right) / 2$, where the parameter $\alpha$ described in Section 4 was chosen to minimise $\sigma_{y}^{\mathrm{p}}$ or $\sigma_{y}^{\mathrm{f}}$ as appropriate.

The values of $\sigma_{y}^{\mathrm{p}}, \sigma_{y}^{\mathrm{f}}$, and the analogous quantities for the horizontal plane $\sigma_{x}^{\mathrm{p}}$ and $\sigma_{x}^{\mathrm{f}}$, corresponding to each of these three methods are given in Table 2 for each experiment. At L3, there were long term stability problems with the BOM electronics gain calibrations, which resulted in it not being meaningful to quote resolutions on a 'per period' basis. For L3 and OPAL, no measurements of QS0 movements were available to correct the BOM data, whilst for ALEPH and DELPHI the potentiometer systems were used to derive the results given in the tables. The hydrostatic system at DELPHI gave results consistent with those from the potentiometers. The corresponding values of $\alpha$, derived by minimising the values of $\sigma_{y}^{\mathrm{p}}$ or $\sigma_{y}^{\mathrm{f}}$, are given in Table 3 .

\subsection{Discussion}

The results shown in table 2 show considerable similarities between the experiments. In both coordinates, the resolution of the 'constant assumption' which is 
a measure of the RMS beam spot movement at the IP, is much better when fill by fill offset subtraction is used. This shows that substantial beam spot movement occurs from one fill to the next, and that it is usually relatively stable within individual fills. When the BOM system is used without fill by fill offset subtraction in ALEPH, DELPHI and OPAL, the resolution is improved considerably in both $x$ and $y$, but by different amounts in each experiment. The BOMs are therefore tracking the between-fill movements of the beam spot position, but the precision is limited by the long term stability of the BOM gain calibrations.

The performance of the BOM system within each fill is more difficult to quantify, since the beam spot is itself rather stable in most fills, and correspondingly little BOM measurement movement is seen. However, in some fills large beam spot movements are seen, which are correctly tracked by the BOMs (see Figs. 5 and 6).

In ALEPH and DELPHI, further improvements in the vertical BOM resolution result when the QS0 measurements are used to correct the position derived from the BOMs, according to the formalism of Section 4. Again, the improvements are most visible without fill by fill offset subtraction. Examples of the $y-y^{\mathrm{vtx}}$ distributions in ALEPH from the whole data taking period are shown in Fig. 7, together with the distribution expected from the vertex measurement resolution alone. It can be seen that using the BOM and QS0 measurements successively reduces the width of the distributions and also reduces the non Gaussian tails caused by the small number of fills with large beam spot movement. The best resolutions $\sigma_{y}^{\mathrm{p}}$ and $\sigma_{y}^{\mathrm{f}}$ achieved by ALEPH after QS0 correction appear to be somewhat better than those achieved by DELPHI. However, it should be stressed that the quoted errors are only statistical, and there may also be systematic contributions from the BOM calibration and extrapolation, the QS0 measurements and the estimated vertex beam spot resolution, which may vary between IPs.

The values of $\alpha$ derived by ALEPH and DELPHI (see Table 3) are slightly lower than the 1.4 obtained from the machine optics. This is to be expected, since the QS0 movement is actually measured from surfaces attached to the QS0 support structures beyond the magnets themselves, and so will tend to see a magnified movement. In addition, angular movements of the magnets cannot be excluded, and are not included in the formalism of Section 4. The quoted errors on $\alpha$ are only statistical, and are somewhat smaller for ALEPH than for DELPHI. This is due to the data sample being divided into more 'chunks' and the larger QS0 movements at ALEPH.

In ALEPH and DELPHI, the comparison of the BOM and QS0 measurements on the two sides of the IP, as well as the comparison of these measurements with the vertex detector data, reveals systematic effects that are not visible in Table 2, and appear to be caused by the QS0 motions discussed in Section 5. One typical example from ALEPH is shown in Fig. 8. In this fill, the vertex detector and BOM measurements from left and right sides systematically disagree at the beginning of the fill, and gradually move into agreement after a couple of hours. When corrected for QS0 movements, the left and right BOM data then agree both with each other and with the vertex detector measurements. Another example from DELPHI is shown in Fig. 9. After 5 hours of stable data, the beam spot position as measured by the vertex detector moves by $50 \mu \mathrm{m}$, whilst the BOM measurements remain constant. Again, the discrepancy is tracked by the QS0 


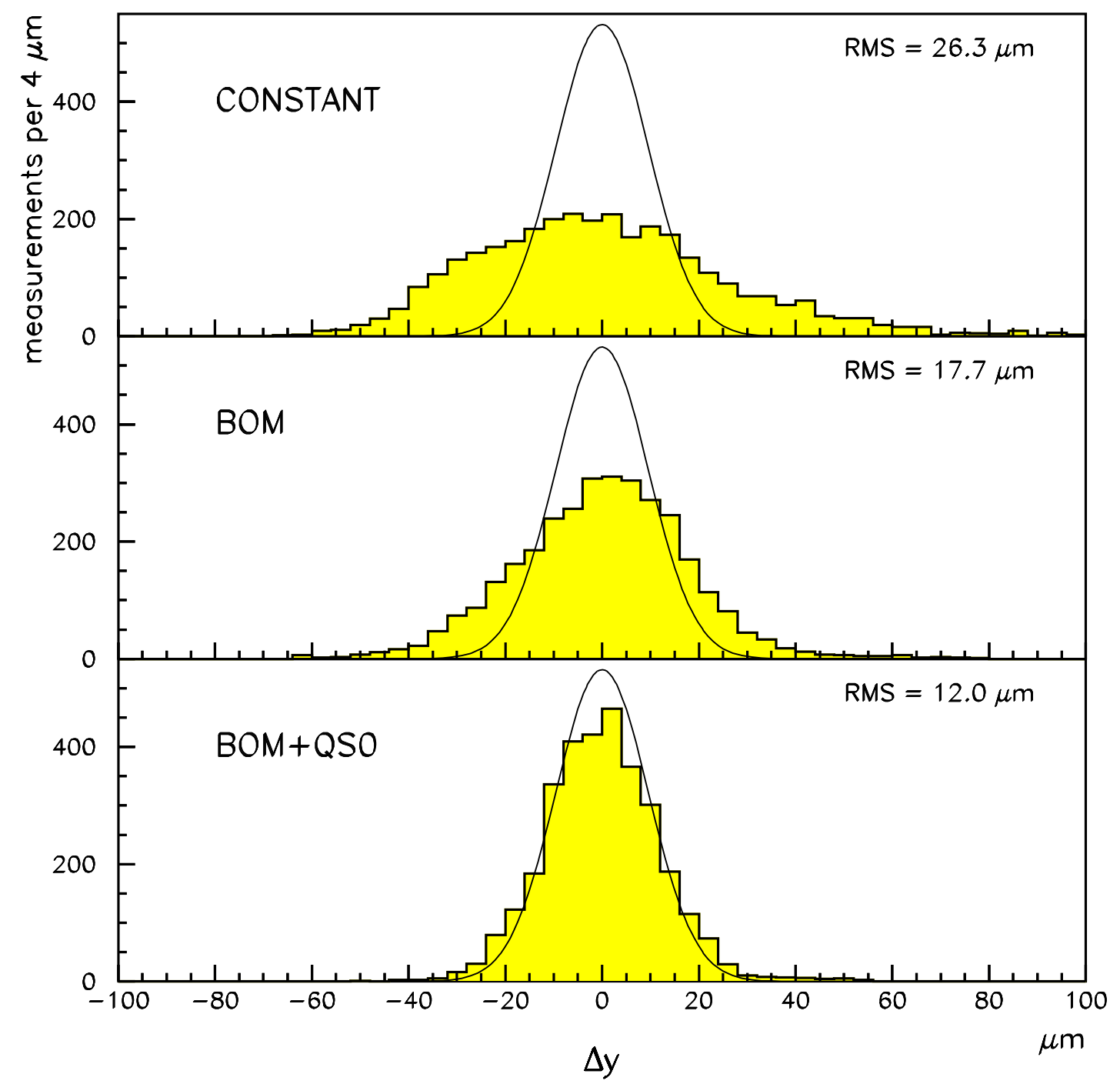

Figure 7: Distributions of $y-y^{\text {vtx }}$ (re-centred around zero) obtained in ALEPH from the same set of 3188 measurements $y^{\mathrm{vtx}}$ of the beam spot position between 13th August and 5th October, 1995. They correspond to $y=0, y=y^{*}$, and $y=y^{*}+1.16\left(y_{L}+y_{R}\right) / 2$, from top to bottom. The curve is a Gaussian distribution (normalised to the other distributions) with an RMS equal to $\sigma_{y}^{\text {vtx }}=9.6 \mu \mathrm{m}$, the mean uncertainty on $y^{v \mathrm{vx}}$. The improvement in beam spot resolution and reduction of non-Gaussian tails resulting from the use of BOM and QS0 data can be clearly seen

motion, and agreement is restored once the BOM measurement is corrected.

In both cases, the QS0 movements are thought to be of thermal origin, with time constants of around 1-2 hours. In the first case, the ramping up of QS0 current and subsequent change in heating effect in the QS0 magnet and its support structure causes QS0 movement which is still happening at the beginning of physics collisions. The second case occurs during stable running, and is thought to be due to external thermal influences. For example, during one fill in 1995 the 


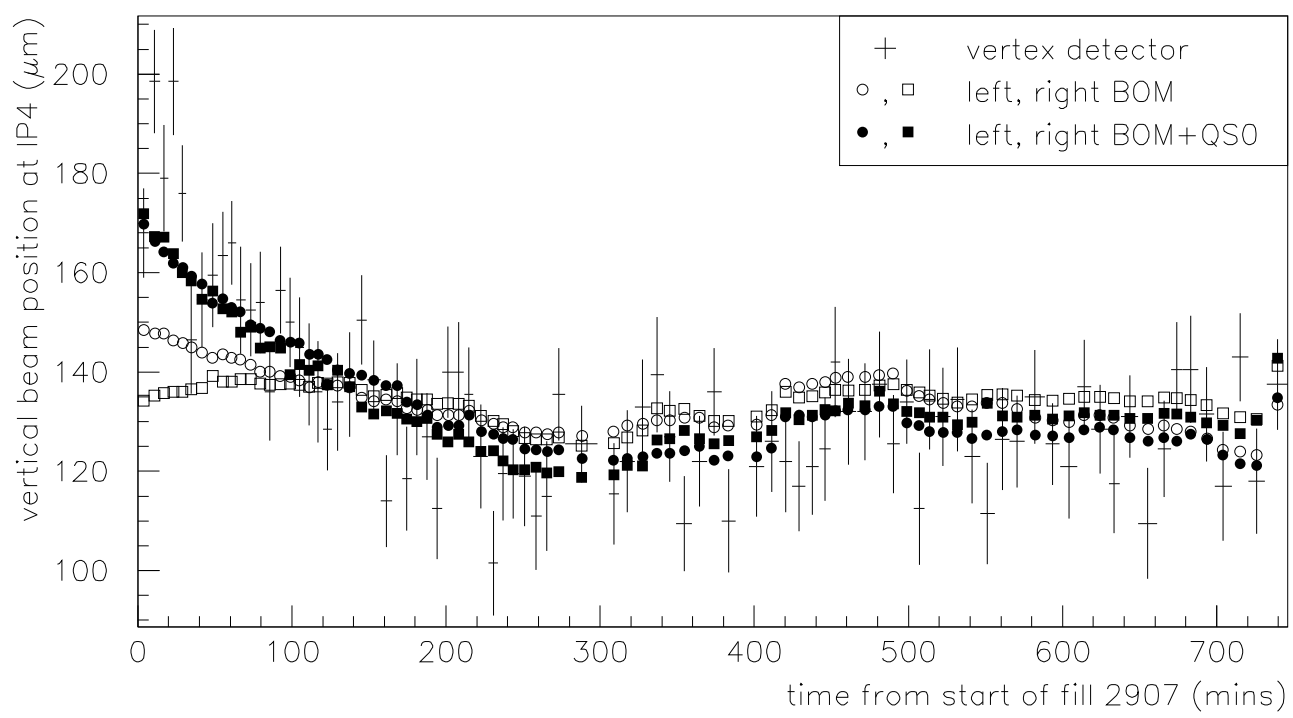

Figure 8: Beam spot measurements at IP4 (ALEPH) for fill 2907, showing vertex detector (crosses), BOM (open symbols) and BOM corrected for QS0 motion (filled symbols). The measurements obtained from the data on the left (right) side of IP4 are shown as circles (squares). The vertical motion of the right side QS0 magnet in fill 2907 is shown in Fig. 3.

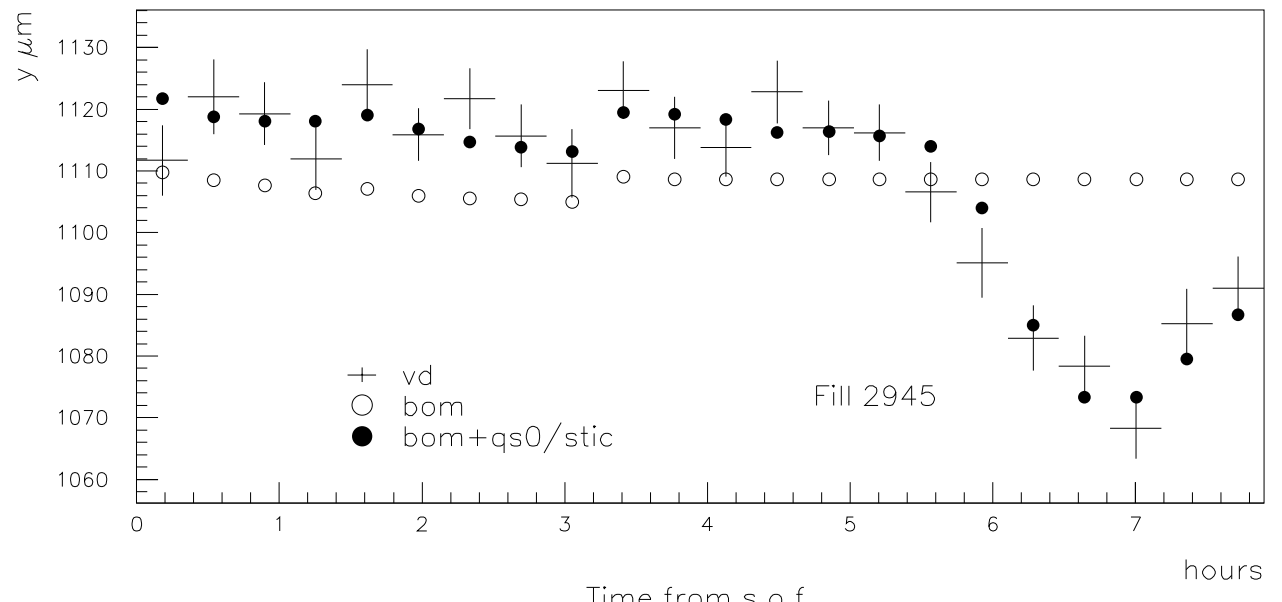

Figure 9: Beam spot measurements at IP8 (DELPHI) for one fill, showing vertex detector (crosses), BOM (open circles) and BOM corrected for QS0 motion using pin potentiometer probes (filled circles). 
air-conditioning in the ALEPH cavern failed, causing QS0 motion and a discrepancy between the BOM and vertex measurements, which was removed when the QS0 correction was applied.

The horizontal beam spot motion and BOM resolution have also been studied using the same formalism, and the results are given in Table 2. As expected from the focusing factors, the BOM resolution is considerably worse than in the vertical plane. The pin potentiometer probe systems in ALEPH and DELPHI also measure in $x$, and significant movement is seen. However, this motion appears to occur on much shorter time scales than in $y$, and no resolution improvements were found using QS0 corrections in $x$. The beam is focused in the horizontal plane by quadrupoles further from the IP (principally QS1), and so any movement of these magnets will have a much larger effect than horizontal movements of QS0. But due to the size of the luminous region in $x(100-150 \mu \mathrm{m})$, a very accurate measurement in the horizontal plane is not useful, and consequently position monitoring of QS1 and other magnets has not been installed.

\section{System performance at higher energies}

The short run of the LEP collider at $\sqrt{s}=130-140 \mathrm{GeV}$ in November 1995 produced about $5 \mathrm{pb}^{-1}$ of data per experiment, and provided a first opportunity to demonstrate the derivation of beam spot information in conditions similar to those at LEP2. Conversely, it was no longer possible to cross-check the results accurately using vertex detector information.

\subsection{Measurements using tracks}

As explained in Section 2.3, the largest rate of tracks at energies above the $Z^{0}$ resonance comes from $\gamma \gamma$ events. These tracks are predominantly low $p_{T}$ and distributed towards the forward angular region. Similar algorithms to those used at LEP1, but with relaxed track selection criteria, were employed to monitor the beam spot position, and the results are listed in Table 4 . The estimated resolutions differ significantly between experiments, due not only to the different averaging times used, but also to the different triggering and track selection requirements (1

or 2 reconstructed tracks per event, different momentum, transverse momentum and angular cuts). However, it is clear that the tracking beam spots do not provide sufficient information to monitor the beam spot to a precision of $\sigma_{y}<20 \mu \mathrm{m}$ on timescales of a few minutes.

\subsection{Measurements using the BOM system}

The data from the BOM system have been analysed using the same method as in Section 6, and the available results are listed in Table 5. The BOM system was not working at the beginning of this rather short period, and there were various other problems, so it was not possible to do a complete analysis in all experiments. At IP8 (DELPHI), the horizontal BOM information was found not to be usable, so no analysis was attempted for the horizontal plane. ALEPH recalculated the value of $\alpha$ using the LEP1.5 data, and got consistent results compared to that 


\begin{tabular}{|c|c|c|c|}
\hline Experiment & Mean time & \multicolumn{2}{|c|}{ Average resolution } \\
& interval $($ mins $)$ & $\sigma_{y}^{\text {vtx }}(\mu \mathrm{m})$ & $\sigma_{x}^{\text {vtx }}(\mu \mathrm{m})$ \\
\hline \hline ALEPH & 5 & 26.1 & 28.3 \\
DELPHI & 20 & 19.8 & 40.8 \\
L3 & 43 & 26.9 & 27.6 \\
OPAL & 15 & 20.6 & 33.4 \\
\hline
\end{tabular}

Table 4: Summary of the average resolutions on beam spot position measurements performed at LEP1.5 with the silicon vertex detectors.

\begin{tabular}{|c|c|c|c|c|c|}
\hline \multirow{2}{*}{ Experiment } & \multirow{2}{*}{ Measurement } & \multicolumn{4}{|c|}{ Resolutions on beam spot position } \\
& & \multicolumn{2}{|c|}{ Vertical plane } & \multicolumn{2}{c|}{ Horizontal plane } \\
& & $\sigma_{y}^{\mathrm{p}}(\mu \mathrm{m})$ & $\sigma_{y}^{\mathrm{f}}(\mu \mathrm{m})$ & $\sigma_{x}^{\mathrm{p}}(\mu \mathrm{m})$ & $\sigma_{x}^{\mathrm{f}}(\mu \mathrm{m})$ \\
\hline \hline & 'constant' & $36.5 \pm 0.9$ & $19.8 \pm 0.9$ & $109.5 \pm 1.9$ & $58.6 \pm 1.2$ \\
ALEPH & BOM & $29.7 \pm 0.8$ & $19.9 \pm 0.9$ & $48.6 \pm 1.0$ & $38.7 \pm 1.9$ \\
(IP4) & BOM+QS0 & $12.7 \pm 1.1$ & $9.0 \pm 1.4$ & $48.4 \pm 1.0$ & $38.6 \pm 0.9$ \\
\hline & 'constant' & $22.5 \pm 0.9$ & $16.4 \pm 0.7$ & - & - \\
DELPHI & BOM & $22.4 \pm 0.9$ & $15.6 \pm 0.6$ & - & - \\
(IP8) & BOM+QS0 & $20.8 \pm 0.9$ & $14.2 \pm 0.6$ & - & - \\
\hline L3 & 'constant' & - & $14.6 \pm 5.2$ & - & $29.4 \pm 6.9$ \\
(IP2) & BOM & - & $15.0 \pm 5.3$ & - & $17.9 \pm 5.6$ \\
\hline OPAL & 'constant' & $31.7 \pm 1.8$ & $22.4 \pm 2.1$ & $51.8 \pm 2.9$ & $27.7 \pm 2.2$ \\
(IP6) & BOM & $21.3 \pm 1.6$ & $12.7 \pm 2.0$ & $23.4 \pm 2.8$ & $15.9 \pm 3.8$ \\
\hline
\end{tabular}

Table 5: Summary of beam spot resolutions measured by the experiments at LEP1.5 (see text). Errors given are statistical.

derived for LEP1, but DELPHI used their LEP1 value as there were not sufficient good BOM data to make an accurate $\alpha$ determination at LEP1.5.

During the LEP1.5 running, the QS0 motion at IP4 was approximately twice that seen at LEP1, consistent with the higher magnet currents required at higher beam energy. However, the QS0s at IP8 (which are of the newer type installed at all IPs for LEP2) did not move any more than during LEP1. Analysis of the orbit corrections required during LEP1.5 fills showed that most of them were caused by motion of the IP4 QS0 magnets.

\subsection{Discussion}

The $y$ resolutions measured at LEP1.5 are worse than those at LEP1, though they show similar trends - the BOM measurements and QS0 corrections produce successively better estimates of the beam spot positions than a constant assumption. The size of the beam spot movement and effect of QS0 corrections are both largest for ALEPH, as expected from the large QS0 movements seen at IP4. The target resolution of $20 \mu \mathrm{m}$ in $y$ has been met on a 'per fill' basis in all experiments, and 
also on a 'per period' basis in ALEPH. The target resolution of $50 \mu \mathrm{m}$ in $x$ has also been met in ALEPH, L3 and OPAL.

The average luminosity during this high energy running was around $1 \times$ $10^{31} \mathrm{~cm}^{-2} \mathrm{~s}^{-1}$. At LEP2, both the luminosity and $\gamma \gamma$ cross section are expected to increase, improving the accuracy of the tracking measurements. This should avoid the need to rely on the long term stability of the BOM measurements, and allow them to be used to follow the beam spot movements within each fill.

\section{Conclusions}

The requirements for beam spot measurements at LEP2 have been studied with Monte Carlo simulations of physics and background processes of interest. These studies show that resolutions of $20 \mu \mathrm{m}$ in $y$ and $50 \mu \mathrm{m}$ in $x$ are adequate, and that higher resolutions do not bring significant additional benefit.

Such resolutions cannot be achieved over short timescales by using tracking measurements alone. The LEP BOM system can achieve the required resolution, and can do even better if the measurements are corrected for the movements of the QS0 focusing magnets. The technique has been studied extensively during the 1995 LEP1 and LEP1.5 running, and compared to the beam spot positions derived from tracking measurements, showing that the resolution target can be met.

Several modifications have been made during the 1995-96 shutdown to improve the accuracy and reliability of the method. The LEP timing distribution system (which caused some periods of lost BOM data) has been upgraded, and new calibration and gain switching schemes have been implemented, which should alleviate the problems seen by some experiments. Pin potentiometer probes have been installed at IP6, the hydrostatic system at IP2 has been upgraded, and new hydrostatic systems have been installed at IP4 and IP6, giving several independent measurements of QS0 position. With these improvements, the beam spot measurements should be reliable and accurate enough to be used in the search for possible new physics at LEP2.

\section{Acknowledgements}

We would like to thank our experimental colleagues and the SL and AT divisions of CERN for making these studies possible. Particular thanks are due to C. Bovet, T. Camporesi, W. Coosemans, M. Jacquet, M. Lamont, W. Murray, J. Pinfold, A. Smith, F. Tecker, and E. Vlasov.

\section{References}

[1] G. Altarelli et al., 'Physics at LEP2', CERN Yellow Report CERN 96-01 vol. 1 page 45 . 
[2] G. Baribaud et al., 'The LEP Beam Orbit Measurement System: Status and Running-in Results', CERN SL/90-31 (BI), Paper presented at the EPAC 90, Nice, France, 1990.

[3] J. Borer et al., 'The Second Generation and Optimised Beam Orbit Measurement (BOM) System of LEP: Hardware and Performance Description', CERN SL/95-60 (BI), paper presented at the Second European Workshop on Beam Diagnostics and Instrumentation for Particle Accelerators (DIPAC 95), Luebeck-Travemuende, Germany, 1995.

[4] C. Bovet et al., 'Luminosity Optimization Using Beam-beam deflections at LEP', paper presented at the EPAC 96, Sitges, Spain, 1996.

[5] H. Grote, C. Iselin, 'The MAD program V8.10', CERN-SL/90-13 Rev. 3 (SL/AP group).

[6] The potentiometer probes were manufactured by Techni Measure (UK). 